Management international

International Management

Gestiòn Internacional

\title{
Achat public et développement durable entre compatibilités et frictions de paradigmes et de pratiques : le cas de l'industrie du transport ferroviaire
}

\section{Public procurement and sustainable development compatibilities and frictions between paradigms and practices: The case of the rail industry}

\section{Compra pública y desarrollo sostenible entre compatibilidades y fricciones de paradigmas y de prácticas: el caso de la industria del transporte ferroviario}

Vincent Helfrich et Fanny Romestant

Volume 20, numéro 1, automne 2015

URI : https://id.erudit.org/iderudit/1045357ar

DOI : https://doi.org/10.7202/1045357ar

Aller au sommaire du numéro

Éditeur(s)

HEC Montréal

Université Paris Dauphine

ISSN

1206-1697 (imprimé)

1918-9222 (numérique)

Découvrir la revue

Citer cet article

Helfrich, V. \& Romestant, F. (2015). Achat public et développement durable entre compatibilités et frictions de paradigmes et de pratiques : le cas de

l'industrie du transport ferroviaire. Management international / International Management / Gestiòn Internacional, 20(1), 78-93.

https://doi.org/10.7202/1045357ar

\section{Résumé de l'article}

Cet article se propose d'analyser, selon deux niveaux de lecture, le degré de compatibilité entre les enjeux développement durable et l'utilisation des achats publics au sein des marchés de l'Union Européenne. Le premier niveau est d'ordre conceptuel, avec un questionnement de la concordance possible entre les principes théoriques, au coeur du développement durable, d'un côté et de l'idéologie européenne, sous-jacente au fonctionnement des achats publics, de l'autre. Le second niveau est d'ordre pragmatique, en interrogeant le degré d'intégration possible du développement durable dans les marchés publics. Nous appuierons nos réflexions sur le cas de l'industrie ferroviaire, à l'aide des matériaux issus d'une recherche-intervention menée au sein d'une entreprise industrielle dans ce secteur.
Tous droits réservés (C) Management international / International Management / Gestión Internacional, 2015
Ce document est protégé par la loi sur le droit d'auteur. L'utilisation des services d’Érudit (y compris la reproduction) est assujettie à sa politique d'utilisation que vous pouvez consulter en ligne. 


\title{
Achat public et développement durable entre compatibilités et frictions de paradigmes et de pratiques : le cas de l'industrie du transport ferroviaire
}

\author{
Public procurement and sustainable development \\ compatibilities and frictions between paradigms \\ and practices: the case of the rail industry
}

\section{Compra pública y desarrollo sostenible entre compatibilidades $\mathbf{y}$ fricciones de paradigmas y de prácticas: el caso de la industria del transporte ferroviario}

\author{
VINCENT HELFRICH \\ Groupe Sup de Co La Rochelle \\ IRIST (Strasbourg)
}

\section{RÉSUMÉ}

Cet article se propose d'analyser, selon deux niveaux de lecture, le degré de compatibilité entre les enjeux développement durable et l'utilisation des achats publics au sein des marchés de l'Union Européenne. Le premier niveau est d'ordre conceptuel, avec un questionnement de la concordance possible entre les principes théoriques, au cœur du développement durable, d'un côté et de l'idéologie européenne, sous-jacente au fonctionnement des achats publics, de l'autre. Le second niveau est d'ordre pragmatique, en interrogeant le degré d'intégration possible du développement durable dans les marchés publics. Nous appuierons nos réflexions sur le cas de l'industrie ferroviaire, à l'aide des matériaux issus d'une recherche-intervention menée au sein d'une entreprise industrielle dans ce secteur.

Mots clés : paradigmes, développement durable, durabilité, achats publics, industrie ferroviaire

\author{
FANNY ROMESTANT \\ Groupe Sup de Co La Rochelle \\ CEREGE (Poitiers)
}

\section{ABSTRACT}

This article analyzes, on two different levels of understanding, the degree of compatibility between sustainable development issues and the use of public procurement in European markets. The first level is conceptual, with a questioning about a possible agreement between the theoretical principles at the heart of sustainable development on the one hand and the European ideology underlying public procurement functioning, on the other. The second level is pragmatic, questioning the possible degree of integration of sustainable development in public procurement. We will support our reflections with the case of the railway industry, using materials from action-research in an industrial company in this sector.

Keywords: paradigms, sustainable development, sustainability, public procurement, rail industry

\section{RESUMEN}

Este artículo pretende analizar, con dos niveles de lectura, el grado de compatibilidad entre los retos del desarrollo sostenible y la utilización de las compras públicas en los mercados de la Unión Europea. El primer nivel es de orden conceptual, con un cuestionamiento sobre la concordancia entre, por un lado, los principios teóricos, eje del desarrollo sostenible y, por el otro, la ideología europea subyacente al funcionamiento de las compras públicas. El segundo nivel es de orden pragmático, cuestionando la posibilidad de integrar el desarrollo sustentable en los mercados públicos. Basaremos nuestra reflexión en el caso de la industria ferroviaria, con la ayuda del material obtenido tras la investigación en una empresa industrial de este sector.

Palabras Claves: paradigmas, desarrollo sostenible, sostenibilidad, contratación pública, industria ferroviaria.
$\mathrm{D}$ epuis plusieurs années, la plupart des gouvernements européens se sont engagés, plus ou moins fortement, en faveur du développement durable. Certaines initiatives liées à ce thème, comme la favorisation des filières courtes, les productions locales ou encore la réduction des émissions de gaz à effet de serre, ont trouvé un certain écho auprès des politiques. Il peut sembler paradoxal, dans ce contexte, de voir que les acteurs publics (collectivités et Etats) n'intègrent pas complétement ces initiatives dans leur propre processus d'achat (marchés publics). On pourrait s'interroger sur les raisons qui empêchent ces organisations d'impulser cette dynamique, en constituant une demande de durabilité dans leurs achats. En réalité, le fonctionnement de ces marchés est conditionné par des procédures très strictes au niveau européen, elles-mêmes basées sur des principes issus de ce que nous proposons de nommer le paradigme européen de l'efficacité économique.

Cet article propose d'établir un lien entre la place accordée à certains postulats économiques dans la construction de la politique industrielle européenne, et sa capacité à favoriser, sur le terrain, des pratiques en lien avec le déve- 
loppement durable. Nous verrons comment une vision dogmatique prend forme dans certains textes fondateurs, afin de constituer ce paradigme européen de l'efficacité économique. Nous verrons, en parallèle, comment ces bases paradigmatiques transparaissent dans certaines formes de durabilités (moyens) qui sont proposées pour atteindre un développement durable (finalité), afin d'identifier les compatibilités et les points de frictions.

L'originalité de notre approche théorique repose sur l'utilisation d'une grille d'analyse basée sur le concept de paradigme scientifique de Thomas Kuhn. Nous montrerons que ce cadre théorique, proposé initialement pour l'étude épistémologique des sciences, peut apporter des clarifications sur le fonctionnement des institutions européennes. Ce dernier nous permet notamment d'éclairer les dynamiques présentes à trois niveaux de notre sujet (dogmatique, législatif et pratique) et leurs interdépendances.

Dans une première partie, nous établirons les bases théoriques du paradigme européen de l'efficacité économique à l'aide de la grille d'analyse des paradigmes de Kuhn. Nous en profiterons pour clarifier les liens entre ce paradigme européen, les formes de durabilités et le concept de développement durable. Dans une seconde partie, nous détaillerons les résultats de notre étude terrain, qui a pour objectif de déterminer quelles sont les compatibilités, les frictions, voire les incompatibilités, entre les enjeux de développement durable et les contraintes de fonctionnement des marchés publics. Cette recherche empirique est composée d'une analyse d'appels d'offres passés dans l'industrie ferroviaire, croisée avec l'étude de documents juridiques et d'entretiens auprès d'acteurs clés de ces marchés. Nous verrons comment ce système est sollicité politiquement pour insuffler des pratiques en lien avec le développement durable, tout en étant fortement conditionné par les exigences européennes de nature paradigmatique.

\section{Le paradigme européen et le développement durable : entre compatibilités et frictions de principes théoriques}

\section{LE CADRE DES PARADIGMES SCIENTIFIQUES} DE THOMAS KuHN

Dans cette recherche, nous mobilisons comme grille de lecture théorique de notre sujet, le concept de paradigme scientifique, développé par Kuhn (1962). En effet, nous pensons que ce concept de philosophie des sciences peut nous aider à porter un regard intéressant et original sur notre problématique.

Le concept de paradigme est défini de multiples façons dans l'œuvre de T. Kuhn, mais nous pouvons le résumer comme un cadre global, partagé de manière tacite par une communauté scientifique, qui permet de définir les théories valides, les façons de poser les problèmes et de les résoudre. On comprend bien que cette notion fait référence à un «savoir tacite», au sens de Polanyi (1958), ce qui la rend de facto difficile à identifier et à restituer. On peut néanmoins la mobiliser pour illustrer une dynamique qui va toucher trois niveaux de notre sujet : les positions dogmatiques des institutions, la forme de ses productions législatives et les pratiques des acteurs sur le terrain (entreprises, consommateurs, collectivités, etc.). Ainsi, l'approche paradigmatique peut constituer une grille d'analyse globale du contexte européen et de ses procédures concrètes, tout comme elle constitue à la fois un apport épistémologique pour l'étude de la construction des sciences et un cadre empirique pour l'étude des conséquences de cette construction sur la vie de laboratoire.

Les précisions faites par T. Kuhn sur ses propres travaux peuvent aussi être mobilisées pour analyser les paradigmes étudiés ci-après. Par exemple, la notion de «matrice disciplinaire ${ }^{1}$ ", introduite dans la postface à partir de la réédition de 1969 par Kuhn (2008, pp. 247-255), décrit des variantes plus fines de ce que peut être un paradigme. Nous pouvons ainsi aborder les paradigmes au sens métaphysique, comme modèles heuristiques ou ontologiques qui incitent les acteurs à "adhérer collectivement à certaines croyances" (Kuhn 2008, p. 250) et qui «fournissent au groupe des métaphores et des analogies préférées ou permises [contribuant] ainsi à déterminer ce qui sera accepté comme une explication" (Kuhn 2008, p. 251). Nous pouvons aussi les aborder comme des généralisations symboliques, qui favorisent les "expressions employées sans question ou dissension par les membres du groupe» (Kuhn 2008, p. 248).

Le cadre théorique des paradigmes peut également nous aider à comprendre le lien existant entre le paradigme européen (sphère politique) et le paradigme de l'orthodoxie économique (sphère scientifique). En effet, un paradigme scientifique, notamment en science humaine, se stabilise par un double processus (scientifique ${ }^{2}$ et institutionnel). Le processus institutionnel repose notamment sur une légitimation interne et externe du paradigme scientifique. La légitimation interne fait référence à tous les mécanismes propres à l'institution pour initier ses membres au paradigme et sanctionner positivement leur adhésion au paradigme. La légitimation externe se traduit par une appropriation des productions ou des croyances issues du paradigme par d'autres institutions que la discipline scientifique. Le tableau en annexe 1 reprend les grands éléments des paradigmes de Kuhn que nous mobilisons pour décrire ce que nous proposons de nommer le paradigme européen de l'efficacité économique, son incarnation dans le corpus de textes et dans les pratiques.

1. Cette notion vient compléter la définition du paradigme en la scindant en quatre types : le paradigme comme exemples communs (modèle de résolution d'énigme), le paradigme comme généralisations symboliques, le paradigme comme valeurs et le paradigme comme croyances métaphysiques. Cette distinction apparait suite aux travaux de Masterman (1970) qui avaient critiqué la délimitation sémantique imprécise de la notion de paradigme chez Kuhn, en recensant 22 variantes de définitions dans l'ouvrage de Kuhn de 1962.

2. Le processus scientifique correspond à ce que décrit principalement Kuhn (1962) dans ses travaux (observations, résolution d'énigmes, construction de théories, etc.). Nous ne nous attarderons pas sur ce dernier dans cet article. 


\section{LE PARADIGME EUROPÉEN DE L'EFFICACITÉ ÉCONOMIQUE}

La construction des institutions européennes et de leurs procédures a été marquée par un mélange d'influences historiques, politiques et économiques. Dans un rapport du conseil d'analyse économique sur les politiques de la concurrence, Encaoua et Guesnerie (2006) reviennent sur ces influences qui ont conditionné la construction et l'importance de la politique de la concurrence aux Etats-Unis et en Europe. Ils insistent sur le fait que des deux côtés de l'Atlantique, les émergences de ces politiques répondent à un besoin d'intégration forte des marchés (contexte économique et historique), et que la forme de ces politiques est intimement liée aux travaux des économistes sur ce sujet (contexte théorique) (Encaoua et Guesnerie, 2006, p.33).

Concernant les influences de la théorie économique, bien qu'ils évoquent les différentes nuances ${ }^{4}$ des champs théoriques concernant leurs visions de la concurrence et des préconisations qu'elles engendrent (cf. tableau en annexe 2), on constate que les champs influents sont tous globalement inclus dans le paradigme scientifique de l'orthodoxie économique $^{5}$ qui est plus ou moins proche du courant néoclassique.

Les auteurs de cette étude insistent d'ailleurs sur le fait que les «conceptions dominantes [...] ont souvent de facto influencé les politiques mises en ouvre» (Encaoua et Guesnerie, 2006, p. 49) et indiquent que d'autres approches plus hétérodoxes, comme celle de Schumpeter (1943) n'ont pas trouvé le même écho auprès des autorités. Nous retrouvons ici un double processus de stabilisation institutionnelle externe (cf. tableau en annexe 1) entre le paradigme de l'orthodoxie économique, d'un côté, dont les fondements trouvent un écho externe dans les institutions européennes, et le renforcement du paradigme européen par des thèses issues du monde académique, de l'autre côté.

Il est aussi intéressant de noter que même les auteurs de cette étude prennent position en faveur des postulats de l'approche orthodoxe en affirmant de manière parfois incantatoire que «la concurrence est non seulement inévitable mais globalement bénéfique» (Encaoua et Guesnerie, 2006, p. 151) et que "la concurrence est une condition nécessaire mais non suffisante pour que l'Union européenne retrouve le chemin de la croissance et de la compétitivité» (Ibid. p. 109). Nous retrouvons ici des signaux qui peuvent être associés au paradigme au sens métaphasique ou symbolique proposé par Kuhn. Cette position illustre, en effet, l'adhésion collective à des croyances, ici le principe d'efficacité (non discuté) de la concurrence de marché (paradigme métaphysique), ce qui engendre une généralisation symbolique où les acteurs vont, par postulat, associer cette concurrence à l'efficacité dans leurs discours.

En plus des influences théoriques synthétisées en annexe 2, le modèle européen a également été fortement influencé par l'Ecole Ordo-libérale allemande. Cette dernière, comme son nom l'indique, défend un modèle libéral mais encadré par de la régulation. Dans ce modèle, la concurrence doit être protégée par la loi pour elle-même (cf. la dernière ligne du tableau en annexe 2).

Au-delà de la politique de la concurrence, c'est bien sur la base de cette vision Ordo-libérale que le Traité de Rome a été modelé. Nous retrouvons d'ailleurs cette idée dans certains discours politiques, à l'image de celui de l'ancien commissaire à la concurrence Karel Van Miert qui indiquait en 1998 que : "C'est donc avant tout à l'Allemagne que l'on doit le fait que la concurrence se soit vu accorder dès le début une importance si grande et qu'elle ait presque joué le rôle de fondement du traité CEE» (Van Miert, 1998). Cette influence est encore présente aujourd'hui dans le Traité consolidé de l'Union Européenne. On y retrouve des références positives à la concurrence tout au long du texte, ainsi qu'un chapitre entier consacré à ce principe. L'article 101 de ce dernier définit d'ailleurs clairement l'orientation européenne : "Sont incompatibles avec le marché intérieur et interdits tout accord entre entreprises, toutes décisions d'associations d'entreprises et toutes pratiques concertées, qui sont susceptibles d'affecter le commerce entre États membres et qui ont pour objet ou pour effet d'empêcher, de restreindre ou de fausser le jeu de la concurrence à l'intérieur du marché intérieur» (UE, 2010b, p. 88). L'article 101 est complété par d'autres, afin de délimiter l'ensemble des pratiques contraires à la concurrence (influence sur les prix, influence sur les conditions des transactions, limitations de productions, répartition de marchés, etc.). On retrouve ici en filigrane le principe d'une autorégulation du marché par lequel les acteurs n'ont pas de pouvoir d'influence $^{6}$ De plus, l'article 106 du même chapitre étend l'application de ces obligations aux différentes organisations publiques, ce qui est cohérent avec un rejet de l'interventionnisme étatique sur le marché ${ }^{7}$ Cet article 106 va notamment influencer la nature des règles de fonctionnement des marchés publics qui feront l'objet de la seconde partie de notre article.

Cette validation constitutionnelle de la concurrence, comme situation optimale, se décline finalement dans tout le corpus des productions européennes (cf. figure 1). Ces généralisations symboliques, selon la terminologie des paradigmes de Kuhn, sont ainsi présentes dans des textes fon-

4. On peut évoquer la nuance entre la défense de la structure du marché (défendre l'atomicité) et la lutte contre le pouvoir de marché (position dominante). Dans le second cas, un oligopole peut être une situation concurrentielle si aucun pouvoir de marché ne se dégage. C’est un principe au cœur de la théorie des marchés contestables (Baumol, 1982).

5. L'orthodoxie économique caractérise bien un paradigme scientifique au sens de Kuhn, qui admet pour postulat de base l'individualisme méthodologique associé à un contexte de rareté des ressources. La combinaison de ces deux éléments conduit à de grandes hypothèses de base comme les comportements normalisés (modélisables); la reconnaissance du marché comme lieu unique de l'échange optimal (terrain d'étude restreint); ou encore la reconnaissance de l'existence de situations optimales escomptées (équilibres, lois économiques, idéal de la concurrence pure et parfaite, etc.). Ces éléments constituent le socle du paradigme (la manière de poser les problèmes, de résoudre les énigmes, les croyances admises et non-rediscutées, etc.).

6. Principe d'atomicité ou au moins du marché contestable.

7. Par contre, dans le paradoxe du modèle Ordo-libéral, il incombe in fine aux Etats et à la Commission d'assurer le respect de ces textes, en intervenant éventuellement auprès des acteurs (entreprises ou Etats) qui pourraient remettre en cause le jeu de la concurrence. 
dateurs (constitution), des textes et procédures juridiques (règlement, jurisprudences) et des textes plus informatifs (communications ou rapport). Ce système de corpus, dont les marchés publics sont emprunts, constitue les bases de ce que nous appelons le paradigme européen de l'efficacité économique.

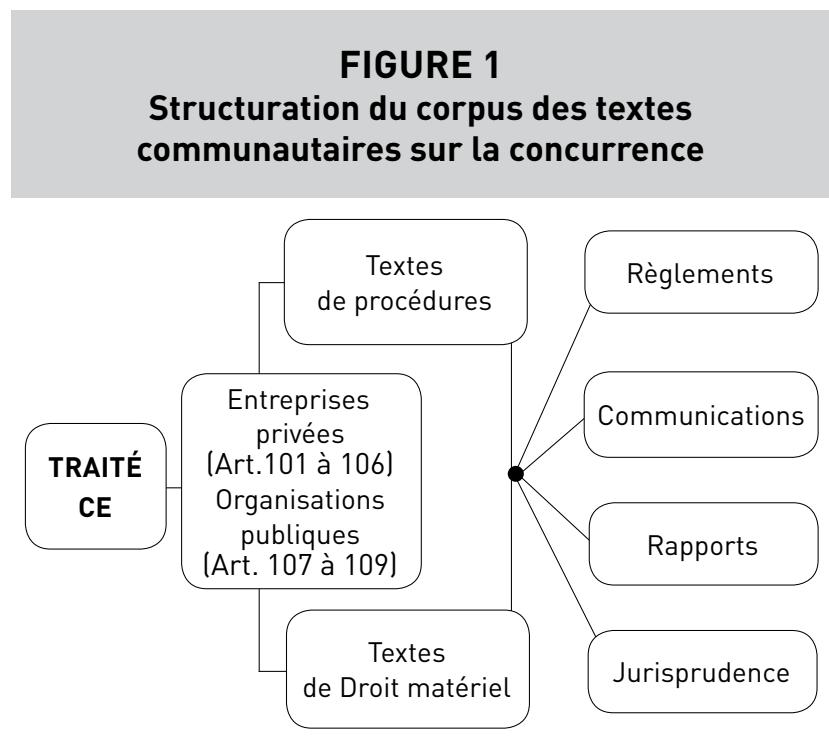

Source : construction des auteurs

Ces éléments sont aussi à rapprocher de notre grille de lecture des paradigmes (cf. tableau en annexe 1), car ils constituent bien des manières de poser les problèmes (dans les textes et les discours) et de les résoudre (dans les procédures). Ces signaux paradigmatiques sont donc présents dans les textes fondateurs du système européen, dans les discours et dans les pratiques.

A l'issue de ce constat, il est maintenant temps de s'interroger sur la compatibilité qui peut exister entre ce paradigme, le développement durable et les formes de durabilités.

\section{DÉVELOPPEMENT DURABLE, DURABILITÉS ET PARAdigme EUROPÉEN : QUELLE COMPATIBILITÉ ?}

Le concept de développement durable a émergé formellement en 1987 avec la publication du célèbre rapport Brundtland. Ce dernier vient se substituer au concept plus ancien d'écodéveloppement qui était axé sur deux piliers (économique et environnemental), en proposant une approche à trois piliers (ajout du pilier social). Le développement durable est défini comme "un développement qui répond aux besoins des générations $d u$ présent sans compromettre la capacité des générations futures à répondre aux leurs» (traduction de UN-WCED (1987, p. 54)).

Selon nous, le développement durable est à considérer davantage comme une sorte d'équilibre de long terme à portée universelle, qui consiste à favoriser le progrès économique et la croissance, en préservant et en valorisant l'environnement, ainsi qu'en respectant l'équité sociale et le bien être humain. Nous distinguerons bien le concept de développement durable, qui ne caractérise pas selon nous un paradigme au sens de Kuhn, des formes de durabilités, qui elles dépendent des positions dogmatiques des paradigmes. Ces dernières constituent en réalité des façons différentes de converger (des moyens) vers le développement durable (une finalité).

D'un point de vue théorique, on retrouve cette variabilité des formes de durabilités dans différents champs théoriques. Cette dernière peut d'ailleurs expliquer le caractère flou que l'on attribut in fine au concept de développement durable. Billaudot et Destais (2009, pp.9-16) proposent une analyse détaillée des différents concepts de durabilité présents dans la littérature économique et dans le champ politique. Cette analyse, résumée dans le tableau en annexe 3, illustre la variabilité des conditions d'un développement durable et de leurs implications normatives.

Nous proposons de synthétiser ces approches dans trois catégories générales au niveau politique (cf. tableau en annexe 4) : une position libérale de durabilité par le marché; une position onusienne de durabilité par un marché régulé et une position de rupture de durabilité par un changement institutionnel fort (économique et politique). Les deux dernières approches ont en commun de suggérer que la mise en place d'un développement durable nécessite au minimum une intervention publique, voire un changement institutionnel significatif selon les visions. Par contre, dans l'approche libérale et le modèle néoclassique, la durabilité serait un état naturel, à condition de laisser le marché fonctionner librement.

On retrouve des typologies similaires sur les formes de durabilités dans la littérature propre au champ du développement durable, qui insistent notamment sur l'opposition entre durabilité forte et faible (Dobson, 1996; Sébastien et Brodhag, 2004; Boutaud, 2005, pp.77-79). Les deux premières approches de l'annexe 4 s'inscrivent davantage dans la durabilité faible et placent la croissance économique au cœur du mécanisme ${ }^{8}$ La troisième approche de durabilité est plus proche de la durabilité forte.

$\mathrm{Au}$ niveau des organisations (publiques ou privées), la contribution pragmatique à un objectif de développement durable va aussi dépendre des différentes formes de durabilités. Dans la position libérale, c'est le marché qui va conduire naturellement les organisations à contribuer à la durabilité, en rendant par exemple les ressources rares plus coûteuses et en incitant à l'innovation. Dans la position onusienne, la contribution des organisations au développement durable pourra prendre la forme d'une démarche de Responsabilité Sociétale (RS), comme l'indique la norme internationale ISO 26000 (ISO 2010), tout en restant compatible avec l'économie de marché. Enfin, dans la position de rupture, l'objet même de l'existence de l'organisation est à repenser totale-

8. Cela correspond, par exemple, à la vision archétypique de l'approche anthropo-centrée du développement durable selon Sébastien et Brodhag (2004), où l'activité économique peut résoudre les problèmes des autres piliers (environnement et social), y compris ceux qui peuvent venir des activités économiques. 
ment autour de l'objectif de développement durable, voire autour d'un objectif de décroissance.

Nous pouvons reprendre l'analyse précédente pour apporter une réponse précise concernant la compatibilité entre le paradigme européen et le développement durable. En effet, le concept de développement durable comme équilibre souhaitable est reconnu de manière consensuelle par l'Union Européenne dans différents textes ${ }^{9}$ Par contre, les formes de durabilités pour atteindre cet équilibre peuvent changer à travers le temps. A ce sujet, Kinderman (2013) met en avant la variation du rôle de la Commission Européenne sur le déploiement de la RS en Europe entre 1993 et 2013. Il souligne dans son travail l'alternance de la stratégie européenne entre une phase socio-libérale (années 1990), une phase néo-libérale (années 2000) et un retour à une phase sociolibérale (années 2010). Le modèle européen a ainsi pu favoriser à la fois une durabilité dans sa forme libérale ou dans sa forme onusienne (selon la typologie de l'annexe 4), ce qui peut vouloir dire que le paradigme européen est compatible avec le développement durable et une partie des formes de durabilités (durabilité faible). En effet, nous avons précédemment constaté que le paradigme européen s'inspire à la fois de l'orthodoxie néoclassique et d'un héritage Ordo-libéral plus interventionniste, ce qui est aussi cohérent avec les conclusions de l'étude de Kinderman (2013). Cela ne permet pas cependant de garantir que ce modèle sera également capable de favoriser la mise en place de stratégies de durabilité forte.

Un des intérêts de notre article est d'aller au-delà de ces débats théoriques. Notre cadre des paradigmes nous a permis, en effet, de comprendre l'articulation entre des positionnements théoriques et politiques, mais il peut maintenant être mobilisé pour comprendre les conséquences sur les comportements pratiques; ce que nous proposons de faire avec l'étude du cas des marchés publics dans l'industrie ferroviaire. Comment ce système est influencé par le paradigme européen? Permet-il de favoriser le développement durable par la commande publique? Comment les acteurs (acheteurs et vendeurs) de ces marchés sont contraints par le paradigme?

\section{L’intégration du développement durable dans les achats publics : entre compatibilités et frictions dans la pratique}

\section{MÉTHOdologIE DE RECHERCHE}

Premièrement, pour traiter du paradigme européen de l'efficacité économique, le choix des marchés publics s'est avéré pertinent dans ce domaine où les acteurs publics vont audelà de leur rôle régalien et d'organisateurs de la vie publique, en entrant dans l'action économique. Ce cadre permet ainsi une mise en tension entre le paradigme de fonctionnement de l'Union Européenne, invoquant une approche libérale de durabilité telle que développée ci-avant, et les enjeux du développement durable.

Deuxièmement, plusieurs raisons nous ont amenés à choisir l'industrie ferroviaire comme terrain d'analyse. D'une part, les enjeux de mobilité sont intrinsèquement liés à des problématiques environnementales (ex. : consommations énergétiques, émissions de gaz à effet de serre) et sociétales (ex. : bruit, aménagement de l'espace, accessibilité pour tous), qui en font une préoccupation des acteurs économiques dans ce contexte. D’autre part, les marchés dans l'industrie ferroviaire sont soumis au cadre réglementaire des marchés publics de par la nature des clients (notamment Etats, collectivités territoriales et entreprises publiques). De plus, il existe de fortes incertitudes sur le sujet des marchés publics durables, tant pour les clients qui doivent rédiger des appels d'offres et n'ont que peu d'outils sur ces sujets ${ }^{10}$ que pour les entreprises candidates aux appels d'offres qui rencontrent des exigences peu claires. Il s'agit, enfin, d'un secteur peu étudié dans la communauté académique, de par sa complexité mais aussi la rareté des données et les difficultés d'accès au terrain. C'est dans le cadre d'une recherche-intervention (Plane, 2000) auprès d'une entreprise européenne du secteur que nous avons pu avoir accès à ce riche terrain, ce qui corrobore l'idée que : "la recherche qualitative est souvent liée à des opportunités, des terrains qui s'ouvrent ou ne s'ouvrent pas» (Dumez, 2013, p.25).

Le présent travail de recherche se veut qualitatif (Miles et Huberman, 2003; Thiétart, 2007, Dumez, 2013), ayant suivi un processus abductif (Dubois et Gadde, 2002) avec des allers-retours constants entre développement du cadre théorique et enquête de terrain. Pour traiter notre problématique et définir comment les enjeux dits « développement durable» se traduisent de manière concrète dans ces marchés, nous avons d'abord réalisé une analyse approfondie des textes législatifs et de la jurisprudence, puis de guides et autres documents de sensibilisation ${ }^{11}$ L'objectif était ici de comprendre ce qui était juridiquement possible de réaliser en matière d'intégration du développement durable dans les marchés publics. Puis, nous avons choisi d'analyser 15 appels d'offres internationaux (liste en annexe 2, tableau 5.1), comprenant principalement les documents type CCTP, CCAG, CCAP, et $\mathrm{RC}^{12}$ Nous avons ainsi pu identifier, par un codage thématique manuel, la nature des exigences environnementales, sociales et plus largement sociétales exprimées dans les documents de consultation. Une première liste d'exigences

9. Voir les communications de la Commission Européenne : UE (2001a, 2002, 2010a, 2011a, 2011b).

10. Les guides ou méthodes développés spécifiquement sur les achats publics durables (Exemple : Guides de l'ADEME, guide de la DAJ -Direction des Affaires Juridiques du Ministère de l'Economie et des Finances-, etc.) concernent souvent des achats courants (textile, restauration collective, etc.) mais rarement des achats stratégiques (tels que les projets de transport ou encore d'infrastructures).

11. Ex. European Union (2011) Buying green, a handbook on green public procurement, 2nd Edition, Luxembourg: Publications Office of the European Union, 2011.

12. CCTP (Cahier des Clauses Techniques Particulières) : ce document décrit les exigences (spécifications techniques et conditions d'exécution) du marché, c'est-à-dire les dispositions techniques nécessaires à l'exécution des prestations du marché. Le CCAG (Cahier des Clauses Administratives Générales) et le CCAP (Cahier des Clauses Administratives Particulières) déterminent les conditions dans lesquelles les marchés sont exécutés. Le RC (Règlement de la Consultation) fixe les règles particulières de la consultation (caractéristiques principales de la procédure et du choix de l'offre). 
environnementales (ex. "déterminer le niveau de consommation énergétique des matériels roulants») a été réalisée, exigences qui ont ensuite été regroupées par thème (exemple pour la catégorie environnement : Energie). Enfin, nous avons poursuivi par une démarche de recherche qualitative avec trois séries d'entretiens semi-directifs (liste en annexe 5, tableau 5.2 et 5.3) d'experts (19), de clients (16) et d'autres parties prenantes (16), ayant pour objectif de comprendre les subtilités dans l'opérationnalisation des réglementations marchés publics relatifs à des projets de transports et concernant des enjeux de développement durable. Des guides d'entretien spécifiques suivant la nature des interviewés ont été élaborés, de manière à interroger les acteurs selon leur sphère d'influence. L'analyse des entretiens a repris les thèmes et sous-thèmes identifiés au sein de l'analyse des documents d'appels d'offres pour en extraire les compatibilités et points de friction.

\section{LE SYSTÈME DES ACHATS PUBLICS ET L'ÉVOLUTION DU CADRE INSTITUTIONNEL EUROPÉEN}

La finalité de la réglementation européenne en matière de marchés publics est d'établir des règles de mise en concurrence des entreprises en respectant les principes de non-discrimination, de liberté d'accès, d'égalité de traitement, de transparence et de proportionnalité. Ces règles représentent typiquement une mise en application de certains principes de la "concurrence pure et parfaite», en cohérence avec le paradigme évoqué plus haut. Dans le champ du marketing industriel, Cova et al. (1992), parlent de «dogme» de la vision néo-classique dans les appels d'offres. Viau (2003) complète cette idée : «ce modèle [le processus d'achat public] s'inscrit a priori dans le paradigme concurrentiel, fondé sur l'étude de transactions économiques obéissant aux lois d'un marché pur et parfait». Cova et al. (1992) mettent cependant en évidence certaines frictions entre le socle réglementaire érigé par les institutions européennes en matière de commande publique et le "chaos» de la réalité des projets. En effet, au regard du socle réglementaire, la méthode de détermination du prix idéal reste in fine la plus importante à maîtriser pour les candidats. Dans la pratique, non seulement le choix des attributaires peut ne pas s'avérer si équitable que souhaité (asymétrie d'information, délicate comparabilité des offres, etc.), et les entreprises peuvent mettre en place des stratégies de soumission leur étant favorables (ex. stratégies d'offres créatrices de projets). Dans un tel contexte, on peut se demander quelle place peuvent prendre les enjeux de développement durable dans ce système.

La première directive européenne sur les marchés publics remonte à 1971 (marchés de travaux). A cette époque et jusqu'aux directives de 2004, aucune référence explicite à l'environnement ou aux conditions sociales de production sont données, nonobstant les diverses décisions de la Cour de Justice de l'Union Européenne ${ }^{13}$ Le Traité d'Amsterdam en 1997 va renforcer la prise en compte de l'environne- ment dans les politiques européennes. Dès lors, la philosophie générale européenne qui préside toute réflexion en la matière, est que la croissance est nécessaire pour parvenir aux objectifs du développement durable et qu'il n'existe pas de contradiction fondamentale entre croissance et qualité environnementale. Il faut alors davantage rechercher une synergie entre les deux; les réglementations concernant les marchés publics renvoient à ce paradigme ${ }^{14}$ qui s'inspire de l'approche de durabilité faible (Dobson, 1996), en cohérence avec nos observations précédentes sur le paradigme européen.

Lactualité européenne en matière de régulation converge cependant vers les signaux faibles d'une évolution des textes en faveur des enjeux du développement durable. La réciprocité dans le traitement des sujets montre la volonté de la Commission Européenne d'aller dans le sens d'une meilleure prise en compte des enjeux sociétaux dans les marchés publics. D'un côté, le développement durable est présenté comme le second enjeu de la révision des directives marchés publics, comme le souligne Michel Barnier, Commissaire Européen chargé du marché intérieur et des services : "Cette réforme permettra par ailleurs une meilleure qualification des marchés publics à travers la prise en compte d'objectifs sociétaux qu'il s'agisse de l'environnement ou l'intégration sociale. Cela se traduit notamment par l'introduction d'un concept $d u$ coût du cycle de vie et la possibilité donnée aux acheteurs publics de prendre en compte le processus spécifique de production des biens, travaux et services achetés dans le choix de l'attributaire du contrat.» (UE, 2013). De l'autre, les marchés publics sont présentés comme un levier d'action vers le développement durable dans les textes de la Commission Européenne sur la RSE (UE, 2011, p. 13). Les institutions européennes reconnaissent depuis peu de temps le potentiel dans l'utilisation stratégique des marchés publics comme levier de la stratégie Europe 2020 (UE, 2010a), les marchés publics représentant près de $19 \%$ du PIB européen. Non seulement, les pouvoirs publics peuvent contribuer à travers leur pouvoir d'achat au développement durable, mais ils peuvent aussi avoir un rôle de démultiplicateur en incitant les acteurs de la supply chain à développer des produits et services durables. On aperçoit ainsi ici le début d'une intervention institutionnelle en faveur du développement durable, cohérente avec les observations de Kinderman (2013) sur un retour à une approche socio-libérale depuis les années 2010.

Cette évolution récente de l'Europe en la matière n'est ainsi pas anodine et renforce la nécessité d'une meilleure compréhension des évolutions sous-jacentes au socle réglementaire des marchés publics. Cependant, compte tenu des propriétés du paradigme européen et de ses visions de durabilité évoquées précédemment, et au regard de la quête contre le protectionnisme prégnante dans les fondements de l'Union Européenne, des points de frictions émergent tout

13. Ex. : Arrêt du 26 septembre 2000 de la CJCE (région Nord-Pas-de-Calais et le Département du Nord contre Commission), Arrêt Concordia Bus Finland Oy Ab de la CJCE en date du 17 septembre 2002, Arrêt Wienstrom de la CJCE en date du 4 décembre 2003, etc.

$14 \mathrm{UE}(2001 \mathrm{~b})$. 
de même dans la diffusion du développement durable dans les marchés publics.

\section{RÉSULTATS EMPIRIQUES SUR LES PRINCIPES LÉGISLATIFS RELATIFS AUX ACHATS PUBLICS DURABLES}

Trois principes sont récurrents dans le fonctionnement des marchés publics. Dans la lignée de ce paradigme économique européen, ils permettent d'assurer l'efficacité de la commande publique. Nous les détaillons à travers quelques exemples en montrant que c'est bien dès ce stade qu'émergent des éléments de friction et de compatibilité relatifs aux enjeux de développement durable.

\section{Principe de non-discrimination}

Il s'agit là du principe de base des marchés publics. La liberté de circulation des biens et des services et l'ouverture des marchés sont le moteur du modèle économique européen néo-libéral. Dans ce cadre, l'efficacité des marchés repose sur une mise en concurrence la plus étendue possible. La nondiscrimination permet ainsi d'acheter efficacement avec le souci permanent d'économiser les deniers publics. Les autres principes qui suivent découlent en partie de ce principe fondamental de non-discrimination.

\section{Exemple de critère exclu pour le choix des soumissionnaires :}

Le choix d'un candidat au regard de sa démarche développement durable ou de sa stratégie RSE car des exigences de ce type risqueraient d'entrainer une liberté de choix inconditionnée de la part du pouvoir adjudicateur. Ainsi, il n'est pas possible d'utiliser la discrimination positive pour valoriser les entreprises engagées en matière de Responsabilité Sociétale.

\section{Liberté d'accès et égalité de traitement des candidats}

Toute entreprise doit pouvoir soumissionner à l'attribution d'un marché. Cela passe par une mise en publicité la plus large possible pour recevoir un maximum d'offres (ou le nombre adéquat selon les procédures) et ainsi choisir la meilleure. Il est strictement interdit de fixer des critères qui entraveraient cette liberté d'accès (ex. utilisation d'un critère de taille de l'entreprise). Cela induit également, pour le pouvoir adjudicateur, l'impossibilité de se fournir systématiquement chez le même fournisseur. Le traitement des offres est strictement réglementé et le favoritisme fait l'objet de sanctions (art. 432-14 du Code pénal français).

Le pouvoir adjudicateur a l'obligation de traiter tous les candidats de la même manière, sur la base de critères établis et "scientifiquement mesurables». Le respect des délais, la délivrance du même niveau d'information pour tous les candidats, des mêmes possibilités de complément de dossiers, etc. sont des conditions à l'égalité de traitement.

D’un point de vue "développement durable», l'établissement de critères scientifiquement mesurables entraîne certaines difficultés : pour preuve, les travaux actuels de la Commission Européenne pour développer des méthodes
Exemple de critère exclu pour le choix des soumissionnaires :

Introduire un critère d'origine des entreprises car celui-ci serait contraire au libre accès pour tous lun des éléments au cœur du traité européen). Il n'est donc pas possible de favoriser des entreprises locales ou nationales (exemple : le fameux «Made in France»).

d'évaluation sur l'analyse de cycle de vie environnementale ou sur le calcul du coût global du cycle de vie.

\section{Transparence}

Afin de garantir la non-discrimination, le pouvoir adjudicateur a pour obligation de veiller à informer de tous les éléments relatifs à la procédure de passation du marché, tout au long du marché : publicité de l'offre, critères de choix des offres, conservation de tous les éléments retraçant les échanges et négociations entre le pouvoir adjudicateur et les entreprises, etc.. Ce principe est aussi un des principes de la Responsabilité Sociétale selon ISO 26000 (ISO, 2010). Il est donc intrinsèquement compatible avec les enjeux du Développement Durable.

\section{Proportionnalité et lien avec l'objet du marché}

Ce principe induit pour les institutions publiques européennes de ne pas dépasser la limite de ce qui est approprié/ nécessaire pour poursuivre l'objectif recherché. En d'autres termes, dans le cadre des marchés publics, le pouvoir adjudicateur n'a pas la possibilité de demander plus que ce qui est en lien avec l'objet du marché (produit/service acheté). Ceci vaut pour des exigences générales sur les entreprises, ou encore pendant le processus d'achat, pour la demande d'informations qui ne seraient pas proportionnées à la finalité de l'acte d'achat.

Sur la notion de proportionnalité, la question de la pondération des critères du jugement est aussi ici en jeu, et fait partie des raisons pour lesquelles les acheteurs restent prudents dans l'utilisation de critères sociaux ou environnementaux (il est recommandé de ne pas dépasser le seuil de $15 \%$ de la note finale pour le jugement des candidats) ${ }^{15}$.

Exemple de critère exclu car non lié à l'objet du marché :

Inciter à l'utilisation de papier recyclé comme fourniture de bureaux des entreprises candidates dans un marché de fourniture de bureau. Cette exigence concerne l'organisation de l'entreprise mais n'est pas spécifiquement liée à l'objet du marché lex. la production du papier en question). Il n'est donc pas possible d'utiliser le marché comme une sphère d'influence (cf. ISO 26000 : ISO 2010) pour diffuser les bonnes pratiques

Exemple de critère exclu car non proportionné à l'objet du marché : Demander la production d'un bilan carbone de la prestation dans le cadre d'un marché de service de prestation intellectuelle. Par contre, ce critère serait pertinent et compatible pour un marché type transport, mais plus controversé dans le cas présenté léventuellement à présenter comme clauses d'exécution du marché).

Exemple de critère autorisé :

Exiger dans les spécifications techniques du produit qu'il soit accessible pour tous (caractéristiques ergonomiques liées au produit). 


\section{FIGURE 2}

Principes de base des marchés publics

Valeurs

(traité Européen)

Principe fondamental

(marchés publics)

Sous-principes

\section{Finalité} poursuivie

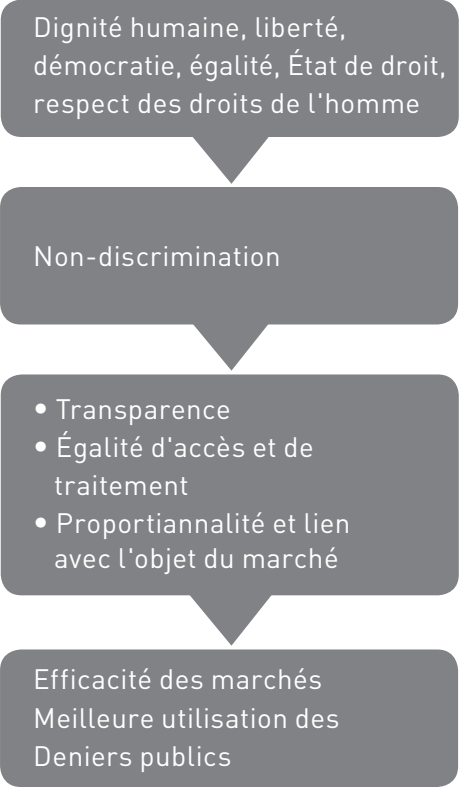

La figure 2 décompose une dynamique en : $1^{\circ}$ ) l'influence du paradigme européen; $2^{\circ}$ ) les principes de base des marchés publics que doivent respecter les acheteurs publics en rédigeant leurs exigences (spécifications techniques, conditions d'exécution) et les critères de jugement des offres; $3^{\circ}$ ) la finalité poursuivie par l'Europe.

Par cette rapide analyse des principes des marchés publics, eux-mêmes issus des grands principes du paradigme européen présenté dans la première partie, nous constatons qu'il existe des points de frictions évidents avec les enjeux du développement durable. Nous observons cependant qu'il subsiste des possibilités de favoriser l'émergence du développement durable avec les marchés publics, mais cela nécessite une véritable expertise dans la rédaction des offres pour ne pas remettre en causes le cadre juridique de ces marchés. Afin de saisir plus précisément ces subtilités et leurs conséquences sur le terrain, nous proposons d'étudier l'intégration du développement durable dans des appels d'offres liés à l'industrie du transport ferroviaire.

\section{RÉSULTATS EMPIRIQUES SUR LES PRATIQUES DES ACTEURS DANS L'INDUSTRIE DU TRANSPORT FERROVIAIRE}

L'industrie ferroviaire concerne les constructeurs de matériels roulants, les équipementiers ferroviaires, les entreprises de la voie et de la signalisation, les ingénieries, mais aussi la réalisation des essais ou encore les métiers du design. Elle représente un marché mondial annuel d'environ 150 milliards d'euros en moyenne sur la période 2011-2013 (UNIFE,
2014), en hausse de $1.5 \%$ par rapport à la période 2009-2011. Cette industrie est décomposée en plusieurs segments dont les services et la production de matériels roulants (trains à grande vitesse, trains régionaux, trains suburbains, métros, tramways, tram-trains, locomotives y compris pour le fret), qui représentent à eux deux la majorité du marché (environ $70 \%)$. L'infrastructure, qui va de la partie génie civile à la pose des rails, est le $3^{\text {ème }}$ segment le plus important $(20 \%$ du marché).

Sur la base de l'analyse des différents matériaux étudiés, nous proposons de mettre en avant quelques exemples représentatifs de thématiques répondant aux enjeux de développement durable selon ses trois piliers. Nous distinguons les thématiques qualifiées de "compatibles" avec le paradigme européen et les marchés publics, et d'autres thématiques entrainant des "points de friction", qui entravent leur mobilisation dans le cadre des marchés publics. Pour les marchés étudiés (voir annexe 5), entre 3 et $20 \%$ des critères de jugement des offres portaient sur des exigences dites «développement durable».

Le tableau 1 présente trois exemples «d'exigences développement durable» compatibles avec les marchés publics.

Concernant le pilier environnemental, l'analyse de cycle de vie (ACV) est totalement cohérente avec le principe du lien avec l'objet du marché, au cœur de la commande publique. Dans tous ses documents sur les marchés publics (dont la récente directive ${ }^{16}$ ), la Commission Européenne privilégie l'approche cycle de vie pour l'évaluation des impacts environnementaux des biens et services achetés. Dans la pratique, les demandes d'ACV dans les documents de consultation sont encore peu fréquentes au regard de la difficulté à en comparer les résultats entre les candidats (en respect du principe de transparence) ainsi que par crainte de limiter la concurrence (et donc l'efficacité du marché). Compte-tenu de l'impulsion donnée par la Commission Européenne, il est probable qu'à l'avenir, ce type d'exigence se développe fortement.

Sur le plan du pilier économique, à l'instar de l'ACV, la Commission Européenne défend fortement l'approche du coût global puisqu'elle est typiquement compatible avec le principe de lien avec l'objet du marché. En revanche, l'utilisation de ce type d'exigence reste encore relativement rare dans les appels d'offres, car (1) les coûts d'investissement et les coûts d'exploitation / maintenance des matériels et infrastructures ferroviaires ne sont pas nécessairement supportés par les mêmes entités dans les collectivités (problème du traçage de l'économie réalisée sur l'utilisation du produit); (2) il y a encore très peu de recul sur certaines catégories de produits (ex. en l'occurrence matériels roulants ayant une longue durée de vie de plus de 30 ans); et (3) le principe d'égalité de traitement reste difficile à respecter dans la mesure où il faudrait proposer une note de calcul extrêmement détaillée aux candidats (ex. note de 


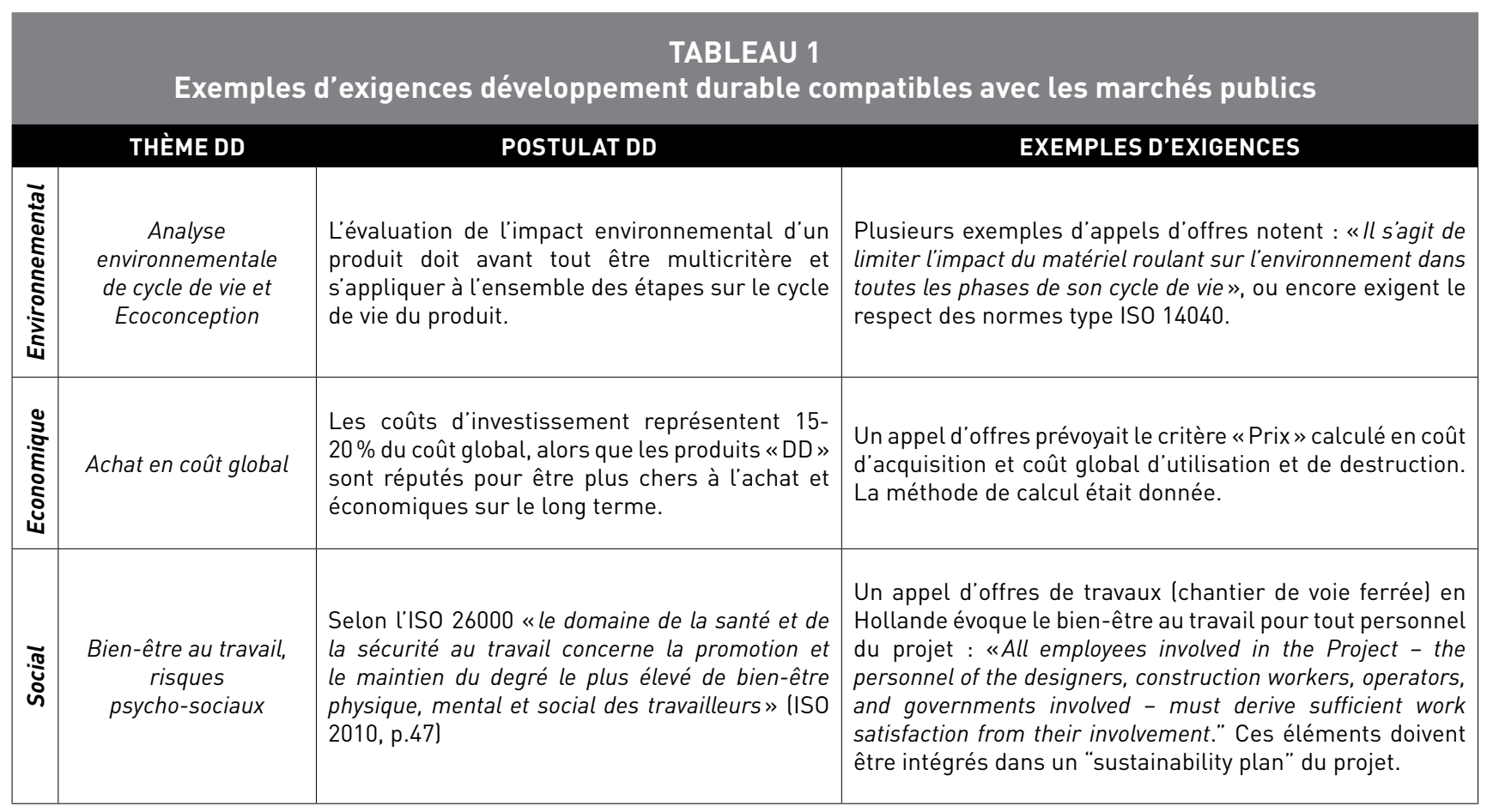

Source : construction des auteurs

calcul pour la maintenance; les offres sont souvent jugées incomparables $\left.{ }^{17}\right)$.

Sur le plan du pilier social, on peut trouver des références compatibles concernant le thème des conditions de travail. Il est encore rare de le trouver dans les appels d'offres (voir dans le tableau 1, le seul exemple dont nous disposons). De plus, il reste uniquement utilisable dans les marchés de travaux et de service.

Le tableau 2 met en avant des critères développement durable qui sont sources de points de friction avec les mar-

\begin{tabular}{|c|c|c|c|}
\hline & THÈME DD & POSTULAT DD & EXEMPLES D'EXIGENCES \\
\hline 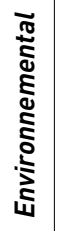 & Changement climatique & $\begin{array}{l}\text { Chaque organisation est responsable de certaines } \\
\text { de ses émissions de CO2, l'acte d'achat peut être un } \\
\text { moyen de réduire indirectement cet impact lacheter } \\
\text { local et logique des circuits courts / toute chose } \\
\text { étant égale par ailleurs). }\end{array}$ & $\begin{array}{l}\text { Fourniture d'un bilan carbone pour la production du } \\
\text { matériel roulant, sur la base de la méthode ADEME. }\end{array}$ \\
\hline 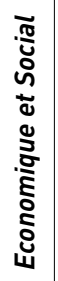 & $\begin{array}{l}\text { Développement } \\
\text { économique local } \\
\text { Ancrage territorial }\end{array}$ & $\begin{array}{l}\text { Acheter local stimule le développement économique } \\
\text { local (création de richesses et d'emplois) } \\
\text { Acheter local stimule le progrès social de la } \\
\text { communauté }\end{array}$ & $\begin{array}{l}\text { Pas d'exigence sur ces thèmes (les clauses } \\
\text { d'insertion de personnes éloignées de l'emploi } \\
\text { dans des marchés de travaux sont le seul moyen de } \\
\text { participer à l'ancrage territorial). }\end{array}$ \\
\hline
\end{tabular}

Source : construction des auteurs

17. Entretien réalisé avec l'ingénieur en charge de l'acquisition du matériel roulant, février 2013 : « Il faut vraiment entrer dans le détail. Certains [constructeurs] donnent des plans de maintenance qui prennent en compte l'opérateur au pied de son organe, qui a déjà ouvert le poussoir ou pas, qui a déjà commencé... Ils ne prennent pas en compte les mêmes hypothèses [pour indiquer] un temps pour une opération. [...]. Après, quand on demande de valoriser, c'est compliqué, on l'a fait. On leur a demandé un plan de maintenance, mais à comparer franchement ... [...]. En plus, on n'est pas sur les mêmes technologies donc on peut avoir un coût de maintenance supérieur d'un constructeur à l'autre, il faut être prudent parce que sur la durée, l'analyse est complexe, franchement.». 
chés publics, notamment parce qu'ils peuvent remettre en cause des principes de base du paradigme européen, en instaurant des barrières à l'entrée ou des discriminations potentielles.

Concernant l'aspect environnemental, il est impossible, dans l'acte d'achat, de valoriser le bilan carbone global d'une entreprise (en référence au principe d'obligation ferme du lien avec l'objet du marché) ni son éloignement de l'entreprise (incompatibilités de principe). Il est en revanche possible de valoriser celui de l'objet du marché, comme dans l'exemple cité. Des frictions existent dans la pratique pour deux raisons principales. (1) Il convient de prendre les précautions d'usage, à savoir préciser la méthode dans les documents de consultation des entreprises (fourniture du cadre de réponse) voire la décrire (éventuellement, mention de la note explicative). Elle ne doit pas non plus porter à confusion (ex. choix du périmètre), sinon le principe de liberté d'accès et de transparence pourraient être remis en cause. Or dans la pratique, il n'y a pas de consensus sur le contenu strict des bilans carbone (ex. problématique de périmètres), les méthodes restant encore flexibles ${ }^{18}$. (2) Il faut être prudent dans l'utilisation du bilan carbone notamment à cause de la disponibilité des données selon le secteur d'activité (faible disponibilité dans le secteur ferroviaire). Compte tenu de ce "clair-obscur», les acteurs interviewés restent réservés sur la demande de bilans carbone associée à des critères de jugement des offres ${ }^{19}$. L'Europe est très attentive à ce point, dans la mesure où il est considéré comme un moyen de pratiquer du localisme ${ }^{20}$. On note en revanche que les suspicions de l'Europe à l'égard du critère $\mathrm{CO} 2$ sont en contradiction avec des enjeux de lutte contre le changement climatique. Si certains paradoxes persistent entre politiques publiques et marchés publics (ex. changement climatique), des avancées de la jurisprudence (notamment l'évolution du lien avec l'objet du marché dans l'arrêt Derichebourg du 15 février 2013) (Conseil d'Etat 2013), le perfectionnement des méthodes d'évaluation des bilans carbone ainsi que l'évolution des compétences de part et d'autre, montrent que le critère $\mathrm{CO} 2$ pourrait prendre de l'importance à l'avenir dans les marchés ferroviaires.

Concernant l'aspect social, il est intéressant de souligner que l'exemple du tableau 1 (Bien-être au travail, risques psycho-sociaux) deviendrait totalement incompatible dans le cadre d'un marché de fourniture (ex. production de matériels roulants). L'acheteur public n'a pas en effet la possibilité d'insérer des exigences relatives à la politique sociale de l'entreprise (incompatibilité de principe). Cette illustration démontre encore la grande subtilité de l'impulsion d'exigences en cohérence avec le développement durable dans les marchés publics, où la même exigence sera compatible ou totalement exclue, selon les marchés. Cet élément est important d'un point de vue opérationnel, tant pour ceux qui commandent ces appels d'offres (collectivités), que ceux qui les rédigent (cabinets spécialisés) et ceux qui y répondent (entreprises). Les entreprises internationales souhaitant candidater sur le sol européen sont d'ailleurs confrontées à ces subtilités qu'il convient d'appréhender pour maximiser leurs capacités à gagner des marchés européens. De même, et alors qu'il s'agit d'une préoccupation majeure de tous les élus interviewés ${ }^{21}$, le thème de l'ancrage territorial (achat local) représente de manière directe une contradiction ferme avec les principes des marchés publics (protectionnisme). Les clauses d'insertion peuvent être utilisées dans les marchés de travaux, mais aucun n'interviewé n'avait entrevu cette clause dans les marchés de fournitures ${ }^{22}$.

Pour finir, nous pouvons ouvrir une discussion d'ordre prospective sur les évolutions du système d'achats publics comme levier de développement durable. En effet, il existe des signaux faibles détectés dans certains appels d'offres et dans certains entretiens. A un niveau procédural, la récente directive européenne sur la passation des marchés publics (UE, 2014) constitue un paradoxe entre d'un côté une impulsion forte en faveur du développement durable ${ }^{23}$ et de l'autre le maintien d'un certain conservatisme ${ }^{24}$. Pour résoudre cer-

18. Entretien réalisé dans le cadre de la recherche-intervention avec le directeur commercial France d'une entreprise du secteur ferroviaire, avril 2012 : "Monsieur [responsable développement durable] m'a souvent dit que quand le client demande un bilan carbone, chacun le fait à sa façon, sur un coin de table, donc les résultats ne veulent rien dire, ils ne sont pas évalués objectivement par les clients et ne sont donc pas valorisés dans la sélection des candidats. Je milite pour avoir des bilans carbone selon des règles identiques pour tout le monde. Pour que les candidats ne puissent pas tricher. Car personne ne vérifie les vrais résultats. Comment les critères sont évalués au stade tendering? Par qui? Comment c'est vérifié en phase de réalisation? Est-ce qu'on peut gagner sur des éléments flous, si oui, c'est scandaleux.".

19. Entretien réalisé avec le directeur du SAE (Service des achats de l'Etat, au sein du Ministère des finances), octobre 2012, en parlant de la valorisation des bilans carbone dans les critères de jugement : "C'est des grands débats, [...] l'acheteur n'étant pas en capacité d'apprécier la meilleure démarche [...], il suffira que quelqu'un lui dise : oui mais le bilan carbone [...] a pris une borne de plus dans l'analyse A, il n'a pas le même périmètre, donc: Terminé. C'est ce qui effraie l'acheteur. ».Entretien réalisé avec le directeur du SAE (Service des achats de l'Etat, au sein du Ministère des finances), octobre 2012, en parlant de la valorisation des bilans carbone dans les critères de jugement : "C'est des grands débats, [...] l'acheteur n'étant pas en capacité d'apprécier la meilleure démarche [...], il suffira que quelqu'un lui dise : oui mais le bilan carbone [...] a pris une borne de plus dans l'analyse A, il n'a pas le même périmètre, donc: Terminé. C'est ce qui effraie l'acheteur.».

20. Entretien réalisé avec la Directrice des marchés publics d'une communauté urbaine française, mai 2013: «Typiquement les circuits courts, par exemple, c'est possible dans l'agroalimentaire à l'heure actuelle; mais ce n'est pas possible ailleurs. On tombe tout de suite sous le coup de la préférence locale...".

21. Entretien réalisé avec l'élu en charge des transports d'une communauté d'agglomération française, février 2013 : «Alors, y compris aussi pour l'approvisionnement des matériaux, on a incité à ce qu'il y ait des préférences locales. Je prends les milliers de mats qui alimentent la ligne aérienne de contact : ça vient de [lieu masqué], donc c'est à 100 et quelques kilomètres d'ici. ».

22. L'insertion par l'activité économique correspond à une action locale de lutte contre le chômage, mais les usines des constructeurs sont rarement localisées dans le bassin local d'emploi (faible intérêt politique)

23. Par exemple, la promotion de la prise en compte du coût global du cycle de vie intégrant l'évaluation des externalités environnementales et sociales. Or, aujourd'hui, il n'existe pas encore de méthodologie d'évaluation utilisable (friction dans la pratique)

24. Par exemple, le lien avec l'objet du marché qui a été conservé ou l'utilisation de formulations souples de type «il convient», qui peuvent être atypiques pour une directive et peuvent rendre les avancées optionnelles. 
taines frictions dans la pratique, les évolutions seront à court et moyen terme davantage orientées sur le développement de méthodologies d'évaluation des impacts environnementaux et sociaux des produits/services tout au long du cycle de vie, qui donneront la possibilité aux acteurs «convaincus» de se saisir du développement durable; à condition de développer les compétences nécessaires des acteurs sur l'ensemble du processus achat (développement d'une véritable ingénierie d'achat conduisant à l'interaction entre les compétences juridiques et techniques). Pour éliminer les incompatibilités soulignées, les évolutions devront être d'ordre paradigmatique, en mobilisant davantage une durabilité forte.

\section{Conclusion}

A travers cet article, nous souhaitions analyser la concrétisation des enjeux de développement durable dans les marchés publics européens, en insistant sur les influences paradigmatiques qu'elle peut subir ${ }^{25}$. Pour ce faire, nous avons restitué les racines théoriques de ce que nous avons appelé le paradigme européen de l'efficacité économique, qui influence en profondeur les procédures des politiques européennes (politique de la concurrence, politique des marchés publics, etc.). Nous avons, en parallèle, évoqué les bases théoriques des différentes formes de durabilités, afin de les confronter avec le paradigme européen, pour en dégager les compatibilités et les points de friction. Ces éléments constituent les deux apports théoriques de notre recherche.

Nous avons également analysé la concrétisation des enjeux de développement durable dans le cadre des marchés publics, et plus précisément dans le domaine de l'industrie ferroviaire. Il est intéressant de noter que certains points de blocage identifiés sont directement liés à des principes de base des marchés publics européens, directement liés au paradigme européen. Dans d'autres régions du monde, les contraintes pesant sur les marchés publics sont parfois moins fortes, en matière de règle de contenu local, par exemple.

La contribution managériale de notre article est notamment basée sur les éclairages qu'il peut apporter aux organisations concernées directement ou indirectement par les marchés publics tant dans le secteur ferroviaire que dans d'autres grands projets (BTP, grandes infrastructures, etc.). Il peut s'agir de rédacteurs d'appels d'offres, de collectivités en situation d'achat, pour qui la mobilisation d'exigences et de critères relatifs au développement durable s'avère encore être un acte risqué (litiges). En effet, une lecture de la situation par le prisme des paradigmes et de leurs évolutions potentielles peut les aider à se prémunir contre ce risque et à exploiter les signaux faibles pour intégrer plus d'exigences en lien avec le développement durable. Dans le champ marketing industriel, une lecture par les paradigmes peut aussi aider les entreprises candidates à des appels d'offres ou les fournisseurs de ces candidats, à anticiper le poids que vont prendre les critères développement durable.
Dans la continuité de notre travail, il serait intéressant d'examiner l'enchainement paradigmatique, législatif et pratique dans d'autres contextes. Nous pensons notamment à des contextes hors de l'Europe, comme la politique de la concurrence américaine qui a subi des influences similaires au cas européen à travers le temps. Cette étude comparée pourrait favoriser la compréhension des écarts qui peuvent exister dans les régulations des achats publics selon les pays (usage de la RSE dans un but protectionniste). Une généralisation de ce travail pourrait aussi passer par une étude dans d'autres industries basées sur la vente de produits complexes, comparables à l'industrie ferroviaire (avec appels d'offres), mais dont les clients ne sont pas concernés par les marchés publics (clients privés), à l'image de l'industrie aéronautique. De même, il serait intéressant d'examiner plus globalement la thématique des achats publics responsables, en abordant également des plus petits projets (fournitures, prestations de services), afin de voir si les compatibilités et les frictions identifiées restent les mêmes.

\section{Bibliographie}

Baumol, W. J. (1982). "Contestable Markets : An Uprising in the Theory of Industry Structure», The American Economic Review, Vol. 72, No. 1, pp. 1-15.

Billaudot, B.; Destais, G. (2009). «Les analyses de la durabilité en économie : fondements théoriques et implications normatives ", Post-Print, HAL. http : //EconPapers.repec.org/RePEc : hal : journl : halshs-00472876.

Boutaud, A. (2005). Le Développement durable : penser le changement ou changer le pansement? Bilan et analyse des outils d'évaluation des politiques publiques locales en matière de développement durable en France: de l'émergence d'un changement dans les modes de faire au défi d'un changement dans les modes de penser. Thèse de doctorat en sciences de la Terre et de l'environnement, École des mines de Saint-Étienne / Université Jean Monnet, Saint-Étienne.

Cova, B.; BAnsard, D.; Flipo, J-P. (1992). «Le marketing de projets : de la réaction à l'anticipation", Recherche et Applications en Marketing, 7(4), 83-104.

Conseil d'Etat (2013). Arrêt Derichebourg Polyurbaine du 15 février 2013, n³63921.

Dobson, A. (1996). "A typology of environmental sustainabilities : An Analysis and a Typology.", Environmental policies, Vol. 5, N.3, pp. 401-428.

Dubois, A.; Gadde L.E. (2002), "Systematic combining : an abductive approach to case research", Journal of Business Research, 55(7), 553-60.

Dumez, H. (2013), Méthodologie de la recherche qualitative : les 10 questions clés de la démarche compréhensive, Paris : Vuibert.

EncaouA, D.; Guesnerie R. (2006). «Politiques de la concurrence ", La Documentation française, collection : Les Rapports du Conseil d'analyse économique.

ISO (2010). Norme ISO 26000 : Lignes directrices relatives à la responsabilité sociétale, ISO Genève.

Kinderman, D. (2013). "Corporate Social Responsibility in the EU, 1993-2013 : Institutional Ambiguity, Economic Crises, 
Business Legitimacy and Bureaucratic Politics», Journal of Common Market Studies, Vol. 51. N 4. pp. 701-720

Kunn, T. (1962). The Structure of Scientific Revolutions, University of Chicago Press, $172 \mathrm{p}$.

Kunn, T. (2008). La structure des révolutions scientifiques, (édition 2008 de l'ouvrage de 1962 avec postface de 1969) Flammarion Champs Sciences, $284 \mathrm{p}$.

Masterman, M. (1970). "The Nature of a Paradigm», dans Lakatos, I. et Musgrave, A. (sous la direction de), Criticism and the Growth of Knowledge, Proceedings of the 1965 International Colloquium in the Philosophy of Science 4 (3 ed.), Cambridge, Cambridge University Press, pp. 59-90.

Miles, M.B.; Huberman A.M. (2003), Analyse des données qualitatives, (M. Hlady-Rispal, tran.), Bruxelles, Belgique : De Boeck Université.

Plane, J.M. (2000), Méthodes de recherche-intervention en management, Paris : Harmattan.

Polanyi, M. (1958). Personal Knowledge, Chicago, The University of Chicago Press, Chicago

Rouveyran, T.; Haouas, I. (2013). «Les critères environnementaux et sociaux dans les marchés publics», Cahiers de droit de l'entreprise, dossier ${ }^{\circ} 5$, septembre-octobre 2013.

Schumpeter, J. (1943). Capitalism, Socialism and Democracy, Londres, Allen Unwin.

Sébastien, L.; Brodhag, C. (2004). «A la recherche de la dimension sociale du développement durable». Revue Développement durable et Territoires, dossier $n^{\circ} 3$.

ThiÉtart, R.A. (2007), Méthodes de recherche en management, Paris : Dunod.

UE (2001a). «Green Paper : Promoting a European framework for Corporate Social Responsibility» COM (2001) 366, Bruxelles, 18 juillet 2001.

UE (2001b). «Communication interprétative de la Commission sur le droit communautaire applicable aux marchés publics et les possibilités d'intégrer des considérations environnementales dans lesdits marchés », COM (2001) 274, Bruxelles, 4 juillet 2001 .

UE (2002). "Communication from the Commission concerning Corporate Social Responsibility : A business contribution to sustainable development», COM (2002)347, Bruxelles, le 2 juillet 2002 .

UE (2010a). «Europe 2020 : Une stratégie pour une croissance intelligente, durable et inclusive», Communication de la Commission, COM (2010) 2020, Bruxelles, le 3 mars 2010.

UE (2010b). «Versions consolidées du traité sur l'Union Européenne et du traité sur le fonctionnement de l'Union Européenne», Union européenne.

UE (2011a). «Communication de la Commission au Parlement Européen, au Conseil, au Comité Economique et Social Européen et au Comité des Régions : Responsabilité Sociale des Entreprises : une nouvelle stratégie de l'UE pour la période 2011-2014», COM (2011) 681, Bruxelles, le 25 octobre 2011

UE (2011b). «Communication de la Commission au Parlement Européen, au Conseil, au Comité Economique et Social Européen et au Comité des Régions : Feuille de route pour une Europe efficace dans l'utilisation des ressources », COM (2011) 571, Bruxelles, 20 septembre 2011.

UE (2013). «Le Commissaire Michel Barnier salue l'accord sur de nouvelles règles en matière de passation de marchés publics» Communiqué de presse de Michel Barnier, MEMO-13-622, Bruxelles, le 26 juin 2013.

UE (2014). Directive 2014/24/UE du Parlement Européen et du Conseil du 26 février 2014 sur la passation des marchés publics, Journal Officiel de l'Union Européenne, le 28 mars 2014.

UNIFE (2014). «World Rail Market Study - Forecast 2014 to 2019», conduit par Roland Berger Consultants, Hamburg, 8 août 2014

UN-WCED (1987). "Our Common Future - report of the World Commission on Environment and Development». Rapport technique, United Nations - World Commissionon Environment and Development.

VAn Miert, K. (1998). L'avenir de la politique de concurrence européenne, Discours prononcé à l'occasion de la remise du prix Ludwig-Erhard, Bonn, 17 septembre. http : //ec.europa. eu/competition/speeches/text/sp1998_042_fr.html.

VIAU, J. (2003). «Pratiques relationnelles et commande publique : enjeux et perspectives». ESKA / Market Management Vol. 3, n², pp. 3-38 


\section{ANNEXES 1 : Grille sur les paradigmes}

\begin{tabular}{|c|c|c|}
\hline $\begin{array}{l}\text { ELÉMENTS DES PARADIGMES } \\
\text { DE KUHN }\end{array}$ & $\begin{array}{l}\text { PARADIGME EUROPÉEN DE L'EFFICACITÉ } \\
\text { ÉCONOMIQUE }\end{array}$ & PARADIGME DE L'ORTHODOXIE ÉCONOMIQUE \\
\hline $\begin{array}{l}\text { Paradigme métaphysique } \\
\text { (croyances admises } \\
\text { et non-rediscutées) }\end{array}$ & $\begin{array}{l}\text { Dogme de l'efficacité des marchés et de la } \\
\text { concurrence. }\end{array}$ & $\begin{array}{l}\text { Postulat du modèle orthodoxe (rationalité, effica- } \\
\text { cité du marché en concurrence). }\end{array}$ \\
\hline $\begin{array}{l}\text { Paradigme comme } \\
\text { généralisation symbolique }\end{array}$ & $\begin{array}{l}\text { - Discours sur la concurrence dans les différents } \\
\text { textes. } \\
\text { - Nature des procédures européennes } \\
\text { (ex : antitrust...). }\end{array}$ & $\begin{array}{l}\text { - Discours sur l'efficacité du marché et de la } \\
\text { concurrence. } \\
\text { - Choix méthodologiques et restrictions de leurs } \\
\text { hypothèses. }\end{array}$ \\
\hline $\begin{array}{l}\text { Stabilisation interne } \\
\text { du paradigme }\end{array}$ & $\begin{array}{l}\text { - Processus institutionnel de diffusion dans le } \\
\text { corpus européen (constitution, réglementation, } \\
\text { communications). } \\
\text { - Initiation au paradigme par la pratique } \\
\text { (procédures). }\end{array}$ & $\begin{array}{l}\text { - Contenu des formations universitaires (initiation } \\
\text { au paradigme). } \\
\text { - Reconnaissance académique des «bons tra- } \\
\text { vaux» (publication, projet de recherche, } \\
\text { récompenses) }\end{array}$ \\
\hline $\begin{array}{l}\text { Stabilisation externe } \\
\text { du paradigme }\end{array}$ & $\begin{array}{l}\text { - Renforcement du paradigme européen par des } \\
\text { thèses issues du monde académique (caution } \\
\text { scientifique à une démarche politique). }\end{array}$ & $\begin{array}{l}\text { - Renforcement du paradigme scientifique par le } \\
\text { soutien des institutions politiques (financement } \\
\text { de recherche, reprises des résultats } \\
\text { de la recherche). }\end{array}$ \\
\hline
\end{tabular}

Source : construction des auteurs

\begin{tabular}{|c|c|c|c|}
\hline \multicolumn{2}{|c|}{ ANNEXE 2 : Influences théoriques de la politique de la concurrence } \\
\hline $\begin{array}{c}\text { COURANTS DE PENSÉES } \\
\text { SUR LA CONCURRENCE }\end{array}$ & $\begin{array}{c}\text { PÉRIODE } \\
\text { D'INFLUENCE }\end{array}$ & $\begin{array}{c}\text { VISION DE LA } \\
\text { CONCURRENCE }\end{array}$ & $\begin{array}{c}\text { NATURE DES INFLUENCES SUR LA } \\
\text { POLITIQUE DE LA CONCURRENCE }\end{array}$ \\
\hline Ecole Structuraliste & 1930's-1970's (USA) & Parfaite latomicitél & $\begin{array}{c}\text { Maintenir une structure de marché } \\
\text { atomisée }\end{array}$ \\
\hline Ecole de Chicago & 1980 's & $\begin{array}{c}\text { Imparfaite } \\
\text { (oligopolistique) }\end{array}$ & $\begin{array}{c}\text { Limiter le pouvoir de marché et privilégier } \\
\text { l'efficacité économique }\end{array}$ \\
\hline $\begin{array}{c}\text { Nouvelle Economie industrielle } \\
\text { (post-Chicago) }\end{array}$ & 1990 's & $\begin{array}{c}\text { Stratégique lthéorie des } \\
\text { jeux) }\end{array}$ & $\begin{array}{c}\text { Analyser les stratégies des firmes } \\
\text { au cas par cas }\end{array}$ \\
\hline Ecole Ordo-libérale allemande & Depuis 1945 (Europe) & Un objet de droit & $\begin{array}{c}\text { Défense de la concurrence en soi } \\
\text { par les Etats }\end{array}$ \\
\hline
\end{tabular}

Source : Synthétisé de Encaoua et Guesnerie (2006, pp.17-54) 


\section{ANNEXE 3 : \\ Le développement durable dans les théories économiques et leurs implications normatives}

\begin{tabular}{|c|c|c|}
\hline CHAMPS THÉORIQUES & CONDITIONS DU DÉVELOPPEMENT DURABLE (DD) & IMPLICATIONS NORMATIVES \\
\hline Néoclassique & $\begin{array}{l}\text { La croissance est le cœur du DD car elle est elle-même durable } \\
\text { (soutenabilité faible / rôle du progrès technique et de la substi- } \\
\text { tuabilité des facteurs / courbe de Kuznets environnementale) }\end{array}$ & Laisser faire le marché. \\
\hline $\begin{array}{l}\text { Economie écologique } \\
\text { (école de Londres) }\end{array}$ & $\begin{array}{l}\text { Concilier l'économie et la préservation de la nature pour } \\
\text { les générations futures (substituabilité limitée des facteurs) }\end{array}$ & $\begin{array}{l}\text { Intervention publique nécessaire } \\
\text { pour internaliser les externalités }\end{array}$ \\
\hline $\begin{array}{l}\text { Economie écologique } \\
\text { (Ecologie industrielle) }\end{array}$ & $\begin{array}{l}\text { Evolution du système industriel copiant la nature lécosystème } \\
\text { de type IIII. }\end{array}$ & $\begin{array}{l}\text { Changement institutionnel auto } \\
\text { réglementé par les entreprises }\end{array}$ \\
\hline $\begin{array}{l}\text { Economie écologique } \\
\text { (école américaine) }\end{array}$ & $\begin{array}{l}\text { Préservation du capital naturel non-substituable } \\
\text { (économie stationnaire) }\end{array}$ & $\begin{array}{l}\text { Nécessité de normes } \\
\text { environnementales }\end{array}$ \\
\hline $\begin{array}{l}\text { Univers controversé } \\
\text { (Incertitude radicale) }\end{array}$ & $\begin{array}{l}\text { Concilier l'économie et la préservation de la nature pour les } \\
\text { générations futures dans un contexte d'incertitudes sur les effets } \\
\text { des actions, les préférences des générations futures, les évolu- } \\
\text { tions de la nature, etc. }\end{array}$ & Intervention publique nécessaire \\
\hline $\begin{array}{l}\text { Univers controversé } \\
\text { (Conventions d'environ- } \\
\text { nement) }\end{array}$ & $\begin{array}{l}\text { Définition du DD issue d'un débat démocratique (recherche de } \\
\text { consensus entre visions différentes) }\end{array}$ & $\begin{array}{l}\text { Analyse ouverte au plan normatif } \\
\text { (besoin de coordination pouvant prendre } \\
\text { plusieurs formes) }\end{array}$ \\
\hline $\begin{array}{l}\text { Analyse globale } \\
\text { (Humaniste) }\end{array}$ & $\begin{array}{l}\text { Conditions normatives définies à l'intersection des } 3 \text { piliers } \\
\text { (économie, social, environnement) }\end{array}$ & Le DD implique d'autres institutions \\
\hline $\begin{array}{l}\text { Analyse globale } \\
\text { (Réaliste ou compréhen- } \\
\text { sive) }\end{array}$ & $\begin{array}{l}\text { Notion positive : compromis historique entre les centres du pou- } \\
\text { voir économique et des forces qui portent les exigences d'équité } \\
\text { et de préservation de la nature. }\end{array}$ & $\begin{array}{l}\text { Analyse ouverte au plan normatif (définit } \\
\text { par le cadre institutionnel en place) }\end{array}$ \\
\hline Décroissance & $\begin{array}{l}\text { Le développement, qui implique la croissance, ne peut être } \\
\text { durable (effet d'entropie) }\end{array}$ & $\begin{array}{l}\text { La décroissance est une nécessité écolo- } \\
\text { gique et humaine }\end{array}$ \\
\hline
\end{tabular}

Source : adapté et complété de Billaudot et Destais (2009, pp.9-16)

\begin{tabular}{|c|c|c|}
\hline \multicolumn{3}{|c|}{ ANNEXE 4 : Les trois formes de durabilité } \\
\hline APPROCHES & DÉFINITION DE LA DURABILITÉ & CONDITIONS DE DURABILITÉ \\
\hline $\begin{array}{l}\text { Position libérale } \\
\text { (orthodoxie économique) }\end{array}$ & $\begin{array}{l}\text { Croissance qui peut durer sans être empêchée par des } \\
\text { contraintes de ressources ou de pollution }\end{array}$ & Marché efficient (en concurrence) \\
\hline $\begin{array}{l}\text { Position progressiste } \\
\text { (onusienne) }\end{array}$ & $\begin{array}{l}\text { Permettre aux générations futures de bénéficier des } \\
\text { progrès dont nous bénéficions actuellement. }\end{array}$ & $\begin{array}{l}\text { Marché ou mode de production capitaliste } \\
\text { (avec plus ou moins de régulation) }\end{array}$ \\
\hline $\begin{array}{l}\text { Position de rupture } \\
\text { (décroissant) }\end{array}$ & $\begin{array}{c}\text { Passe par la décroissance au Nord et un autre type de } \\
\text { vie sociale, renouant avec l'histoire, au Sud }\end{array}$ & $\begin{array}{c}\text { Concevoir de nouvelles institutions politiques } \\
\text { et économiques. Repenser la manière } \\
\text { de mesurer le progrès }\end{array}$ \\
\hline
\end{tabular}

Source : synthétisé de Billaudot et Destais (2009, pp.9-16) 
ANNEXE 5 : Liste des documents et entretiens constituant le matériau de recherche

\begin{tabular}{|l|l|c|}
\hline \multicolumn{2}{|c|}{ TABLEAU 5.1 : Liste des appels d'offres analysés } & 2009 \\
\hline BESANCON (FRANCE) & Matériel roulant (tramway) & 2011 \\
BIRMINGHAM (ANGLETERRE) & Matériel roulant (tramway) & 2009 /2010 \\
BREST (FRANCE) & Infrastructure : Energie traction et basse tension (1ère ligne de tramway) & 2009 \\
BREST-DIJON (FRANCE) & Matériel roulant (tramway) - groupement de commande & 2009 \\
CASABLANCA (MAROC) & Matériel roulant (tramway) & 2011 \\
GRONINGEN (HOLLANDE) & Offre clé en main -DBFMO (Regiotram) & 2011 \\
HYDERABAD (INDE) & Matériel roulant (métro) & 2011 \\
JERNBANEVERKET (NORVEGE) & Signalisation & 2010 \\
LYON (FRANCE) & Matériel roulant (tramway) & 2008 \\
\hline MONTPELLIER (FRANCE) & Matériel roulant (tramway) & 2008 \\
\hline MONTREAL (CANADA) & Matériel roulant (Métro) & 2010 \\
\hline NANTES (FRANCE) & Matériel roulant (tramway) & 2009 \\
\hline PARIS (FRANCE) & Matériel roulant (tramway) & 2009 \\
\hline ROUEN (FRANCE) & Matériel roulant (tramway) & 2009 \\
\hline STOCKHOLM (NORVEGE) & Matériel roulant (train régional) & \\
\hline
\end{tabular}

Source : construction des auteurs

\begin{tabular}{|c|c|}
\hline \multicolumn{2}{|r|}{ TABLEAU 5.2 : Liste des entretiens d'experts réalisés } \\
\hline & ORGANISMES \\
\hline $\begin{array}{l}\text { Experts pour l'axe } \\
\text { «Transport et } \\
\text { développement } \\
\text { durable» }\end{array}$ & $\begin{array}{l}\text { BEI (Banque Européenne d'Investissement), CEREMH (Centre de Ressources \& d'Innovation Mobilité Handicap), CERTU } \\
\text { (Centre d'Etude et de Recherche sur les Transports et l'Urbanisme), Ecole des Ponts et Chaussées - Paris Tech, } \\
\text { FNAUT (Fédération Nationale des Associations d'Usagers des Transports), FNE (France Nature Environnement), GART } \\
\text { (Groupement des Autorités Régulatrices de Transport), LET (Laboratoire d'Economie des Transports de l'Université de } \\
\text { Lyon 2), Commission Transport du Parlement Européen, UNIFE (Union des Industries Ferroviaires Européennes), } \\
\text { Bureau d'étude spécialisé }\end{array}$ \\
\hline $\begin{array}{l}\text { Experts pour l'axe } \\
\text { «Marchés publics } \\
\text { et développement } \\
\text { durable» }\end{array}$ & $\begin{array}{l}\text { AACT (Association des Acheteurs de Collectivités Territoriales), ADEME (Agence de l'Environnement et de la Maitrise de } \\
\text { l'Energie), AFNOR Normalisation, Caisse des Dépôts et Consignations Infrastructures, CDDEEP (Club Développe- } \\
\text { ment Durable des Etablissements et Entreprises Publics, rattaché au MEDDE -Ministère de l'Ecologie, du Développement } \\
\text { Durable et de l'Energie-), Direction Marché Intérieur et Services de la Commission Européenne, OBSAR (OBserva- } \\
\text { toire des Achats Responsables), SAE (Service des achats de l'Etat, au sein du Ministère des finances) }\end{array}$ \\
\hline
\end{tabular}

Source : construction des auteurs

NB. Pour des raisons de confidentialité liée à la politique commerciale de l'entreprise avec laquelle nous menions une recherche-intervention, qui nous a permis ces entretiens, nous ne donnons pas d'indication précise des collectivités et projets en question ici. 


\section{TABLEAU 5.3 : Liste des entretiens d'acteurs réalisés}

\begin{tabular}{|c|c|}
\hline TYPE & INTERVIEWÉS \\
\hline Collectivités et projets étudiés & $\begin{array}{l}3 \text { projets de Tramway ( } 2 \text { nouveaux réseaux et } 1 \text { nouvelle ligne) et } 1 \text { achat de matériel roulant Métro } \\
\text { dans } 4 \text { collectivités françaises ( } 2 \text { communautés d'agglomérations et } 2 \text { communautés urbaines). }\end{array}$ \\
\hline Elus liés aux projets étudiés & $\begin{array}{l}3 \text { élus en charge des transports et déplacements, } 3 \text { élus en charge du développement durable / } \\
\text { écologie urbaine. }\end{array}$ \\
\hline $\begin{array}{l}\text { Responsables techniques des } \\
\text { projets }\end{array}$ & $\begin{array}{l}4 \text { directeurs de la maitrise d'ouvrage, } 4 \text { ingénieurs opérationnels dans le processus d'achat des } \\
\text { matériels roulants ou infrastructures, } 3 \text { directeurs marchés publics / pôle juridique. }\end{array}$ \\
\hline Bureaux d'études & 3 chefs de projet, 2 ingénieurs spécialisés sur le développement durable \\
\hline $\begin{array}{l}\text { Entreprise opératrice du réseau (en } \\
\text { délégation de service public) }\end{array}$ & 3 chefs de projet, 2 responsables qualité, sécurité et environnement et développement durable. \\
\hline Autres parties prenantes & $\begin{array}{l}1 \text { chef de service au Conseil Régional, } 2 \text { présidents d'associations environnementales, } 1 \text { président } \\
\text { d'association de PMR (Personnes à Mobilité Réduite), } 4 \text { présidents/secrétaires généraux d'asso- } \\
\text { ciations d'usagers, } 1 \text { chargé de mission Clauses d'Insertion à la maison de l'emploi, } 1 \text { directeur } \\
\text { régional d'une entreprise partenaire du groupement avec le constructeur ferroviaire, } 3 \text { respon- } \\
\text { sables de département d'organisme d'Etat (VNF, DREAL). }\end{array}$ \\
\hline
\end{tabular}

Source : construction des auteurs

\section{ANNEXE 6 : Synthèse de la recherche}

\begin{tabular}{|l|l|}
\hline $\begin{array}{l}\text { Problématique de la } \\
\text { recherche }\end{array}$ & $\begin{array}{l}\text { Pourquoi les acteurs publics n'intègrent pas complétement les enjeux du développement durable dans les achats } \\
\text { publics? }\end{array}$ \\
\hline Méthodologie & $\begin{array}{l}\text { Recherche qualitative selon un processus abductif : } \\
\text { - Analyse approfondie des textes législatifs, de la jurisprudence, de guides et autres documents de sensibilisation } \\
\text { dans le domaine des marchés publics } \\
\text { - Analyse d'appels d'offres dans le domaine du transport ferroviaire } \\
\text { - Entretiens d'experts et d'acteurs analysés selon un codage thématique manuel }\end{array}$ \\
\hline Apports théoriques & $\begin{array}{l}\text { - Définition d'un paradigme européen de l'efficacité économique } \\
\text { - Analyse des formes et conditions de durabilité } \\
\text { - Triple approche : paradigmatique (dogmatique), législatif et pratique } \\
\text { - Identification des compatibilités, des frictions et des incompatibilités entre achats publics et développement } \\
\text { durable }\end{array}$ \\
\hline Apports managériaux & $\begin{array}{l}\text { - Identification des compatibilités, des frictions et des incompatibilités entre achats publics et développement } \\
\text { durable dans les pratiques } \\
\text { - Contribution de nature procédurale laide au décryptage) pour les organisations concernées directement ou } \\
\text { indirectement par les marchés publics }\end{array}$ \\
\hline
\end{tabular}

